\title{
Forecasting of Chinese Hydropower Generation Using WASD-Neuronet
}

\author{
Yurong Cheng ${ }^{1,2}$, Haiting Ye ${ }^{1}$, Xiaoyu Guo ${ }^{2}$, Chuan $\mathrm{Ma}^{3}$, Bolin Liao ${ }^{3}$, Long Jin*2 \\ ${ }^{1}$ Longdian Branch, Huanghe Hydropower Development Corporation Limited, State Power Investment Corporation Limited, \\ Yongjing, China \\ ${ }^{2}$ School of Information Science and Engineering, Lanzhou University, Lanzhou, China \\ ${ }^{3}$ College of Information Science and Engineering, Jishou University, Jishou, China
}

Received: June 6, 2018

DOI: $10.5430 /$ ijrc.v $1 \mathrm{n} 1 \mathrm{p} 48$
Accepted: August 23, $2018 \quad$ Online Published: August 30, 2018

URL: https://doi.org/10.5430/ijrc.v1n1p48

\begin{abstract}
Hydropower resource is one of the renewable energy sources. With the increasing Chinese economy, people are paying much more attention to sustainable development. The increasing hydropower load is the basis of the development of power industry. Due to the characteristics of electrical energy, predicting the hydropower accurately is a potentially beneficial way to plan hydropower reasonably. This paper presents a neural network method to predict hydropower generation whose data is influenced by several factors such as social economic, population and climate. By using the past 52-year rough data, a 3-layer feedforward neuronet equipped with the weights and structure determination (WASD) method is constructed for the prediction of the Chinese hydropower generation in this paper. By processing mass of data, we could basically predict the hydropower generation using such a WASD neuronet. To a large extent, the trend of developing Chinese hydropower generation in the next years will keep growing.
\end{abstract}

Key Words: Sine neural network (SNN), Weights and structure determination (WASD), Neural network, Hydropower generation

\section{INTRODUCTION}

Environment-friendly requirements about clean and low carbon development become a worldwide and creative topic. Hydropower resource, one of renewable energy sources, has many advantage such as high efficiency, low cost, quick start-up and easy to adjust. In China, there are abundant hydropower resources and hydropower generation is the most important part of energy supply. ${ }^{[1-3]}$ The utilization of water resources will remain in growth in the future according to the relevant policies. Therefore, it is necessary for us to predict the hydropower generation in China. Load forecasting, which is the key component of power system, lays a founda- tion for the plan of expansion, operation and overhaul. ${ }^{[4-6]}$ It can be divided into electric energy forecasting and electric demand forecasting. Electric energy forecasting includes social consumption, net power supply, industry power and regional power, etc. Electric demand forecasting moreover includes maximum load, minimum load, load rate, load curve, etc. For achieving the security and stabilization of power system, Power Dispatching Center distributes the power generation according to different needs. Therefore, forecasting the hydropower generation and predicting it accurately is crucial for the whole power grid.

Nevertheless, many factors may affect the power grid, such

\footnotetext{
*Correspondence: Long Jin; Email: jinlongsysu@foxmail.com; Address: School of Information Science and Engineering, Lanzhou University,
} Lanzhou 730000, China. 
as meteorological conditions, the domestic economy, population, government policy and public emergencies. As a consequence of a large numbers of factors, forecasting the hydropower generation is sufficiently challenging. ${ }^{\text {[1] }}$ Because hydropower generation mainly depends on the inflow water which is strongly associated with the season despite of some small probability events. In this report, the historical data are used to do beneficial research on these contents.

\section{WASD-NEURONET AND THEORETICAL ANALYSIS}

Since 1980s, Artificial Neural Network (ANN) has become the hotspot of Artificial Intelligence (AI) and made great progress in recent years. ${ }^{[8-12]}$ Because of the advantages in ANN, such as nonlinearity, non-convexity, self-adaption, selforganization and self-learning, it becomes an optimal choice to process information in enormous numbers of field. ${ }^{[13-19]}$
ANN has successfully solved many problems in pattern recognition, automatic control, biology, economy and other fields where modern computer is difficult to solve. ${ }^{[20-22]}$ Back propagation (BP) algorithm or support vector machine (SVM) is used widely, however, it is extremely easy to be influenced by the new sample and SVM cannot analyse largescale data precisely. ${ }^{[23-27]}$

We first proposed a single hidden-layer feedforward neural network activated by sine (sine neural network, SNN). Then, a weights and structure determination (WASD) method based on the weights direct determination (WDD) method is used to train the date. ${ }^{[28-31]}$

Therefore WASD method is presented to analyze annual electricity production and a three-layer feedforward neuronet activated by sine is constructed in this paper, of which the model diagram is illustrated in Figure 1.

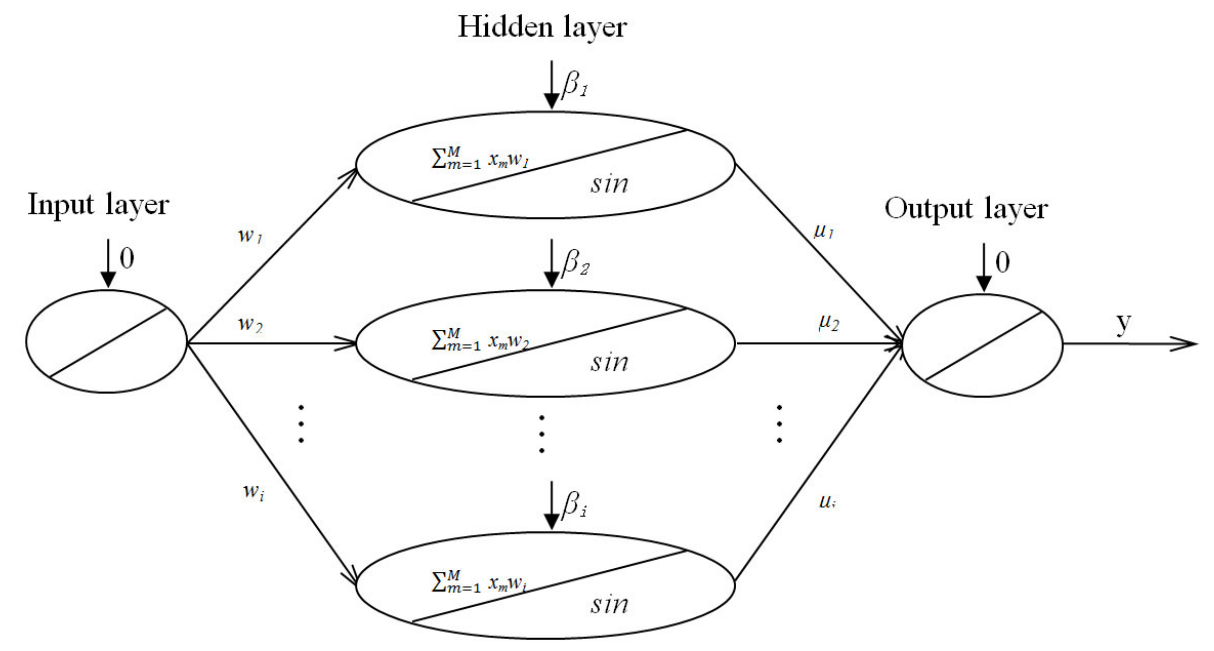

Figure 1. Model of WASD neuronet

Model of the 3-layer feedforward neuronet activated by sine is equipped with the WASD method.

The weights from the input layer to the hidden layer are determined randomly and the weights from the hidden layer to the output layer are calculated by the pseudo-inverse. Denoting $\varphi_{(m, i)}$ as the ith input through hidden layer for getting the exact number:

$$
\varphi_{(m, i)}=\sin \left(\sum_{m=1}^{M} x_{m} w_{i}-\beta_{i}\right)
$$

The output is set as

$$
Y=\mu \varphi
$$

Then, the weights from the hidden layer to the output layer are directly decided by the following formula:

$$
\mu=\varphi^{+} \eta
$$

$\eta$ is the column vectors of the sample (the history data of hydropower generation).

In order to improve the accuracy of prediction, the target output and the network output are required to be analyzed. The mean square error (MSE) is used to point out the differences:

$$
E=\frac{1}{M} \sum_{m=1}^{M}\left(\eta_{m}-\sum_{i=1}^{I} w_{i} \varphi_{(m, i)}\right)^{2}
$$

All data are divided into three parts for learning, verifying 
and predicting respectively. For satisfying more stable and better self-adapted final neuronet, the weighted error is denoted as $E_{w}$, which is a determinant in growth of hidden neuron:

$$
E_{w}=\xi E_{l}+(1-\xi) E_{v}
$$

( $E_{l}$ is the error of the learned data, $E_{v}$ is the error of the verified data and $\xi$ is the proportion of learned data.)

The forecast of Chinese hydropower generation is based on the data from 1952 to 2017 (see Table 1). Due to the rapid development, there is a huge difference in the number of the power generation from 1952 to 2017 . In order to reduce the influence of different number, all the data are normalized into the interval $[-1,1]$. After accomplishing prediction, the figures would be displayed using the raw data.

Table 1. Data for the China's hydropower generation used for analyse and prediction

\begin{tabular}{|c|c|c|c|c|c|c|c|c|}
\hline Year & 1952 & 1957 & 1962 & 1965 & 1970 & 1971 & 1972 & 1973 \\
\hline Data (billion kWh ) & 1.3 & 4.8 & 9.0 & 10.4 & 20.5 & 30.0 & 34.0 & 38.0 \\
\hline Year & 1974 & 1975 & 1976 & 1977 & 1978 & 1979 & 1980 & 1981 \\
\hline Data (billion kWh ) & 43.0 & 45.0 & 45.6 & 47.6 & 44.6 & 50.1 & 58.21 & 65.55 \\
\hline Year & 1982 & 1983 & 1984 & 1985 & 1986 & 1987 & 1988 & 1989 \\
\hline Data (billion kWh ) & 74.4 & 86.36 & 86.78 & 92.37 & 94.53 & 100.01 & 109.15 & 118.4 \\
\hline Year & 1990 & 1991 & 1992 & 1993 & 1994 & 1995 & 1996 & 1997 \\
\hline Data (billion kWh ) & 126.72 & 125.09 & 132.47 & 151.819 & 167.4 & 190.577 & 187.966 & 195.983 \\
\hline Year & 1998 & 1999 & 2000 & 2001 & 2002 & 2003 & 2004 & 2005 \\
\hline Data (billion kWh ) & 208.0 & 203.807 & 222.414 & 277.432 & 287.974 & 283.681 & 353.544 & 397.017 \\
\hline Year & 2006 & 2007 & 2008 & 2009 & 2010 & 2011 & 2012 & 2013 \\
\hline Data (billion kWh ) & 435.786 & 485.264 & 585.187 & 615.64 & 722.17 & 698.95 & 872.11 & 920.29 \\
\hline Year & 2014 & 2015 & 2016 & 2017 & & & & \\
\hline Data (billion kWh ) & $1,064.34$ & $1,130.27$ & $1,051.84$ & $1,081.88$ & & & & \\
\hline
\end{tabular}

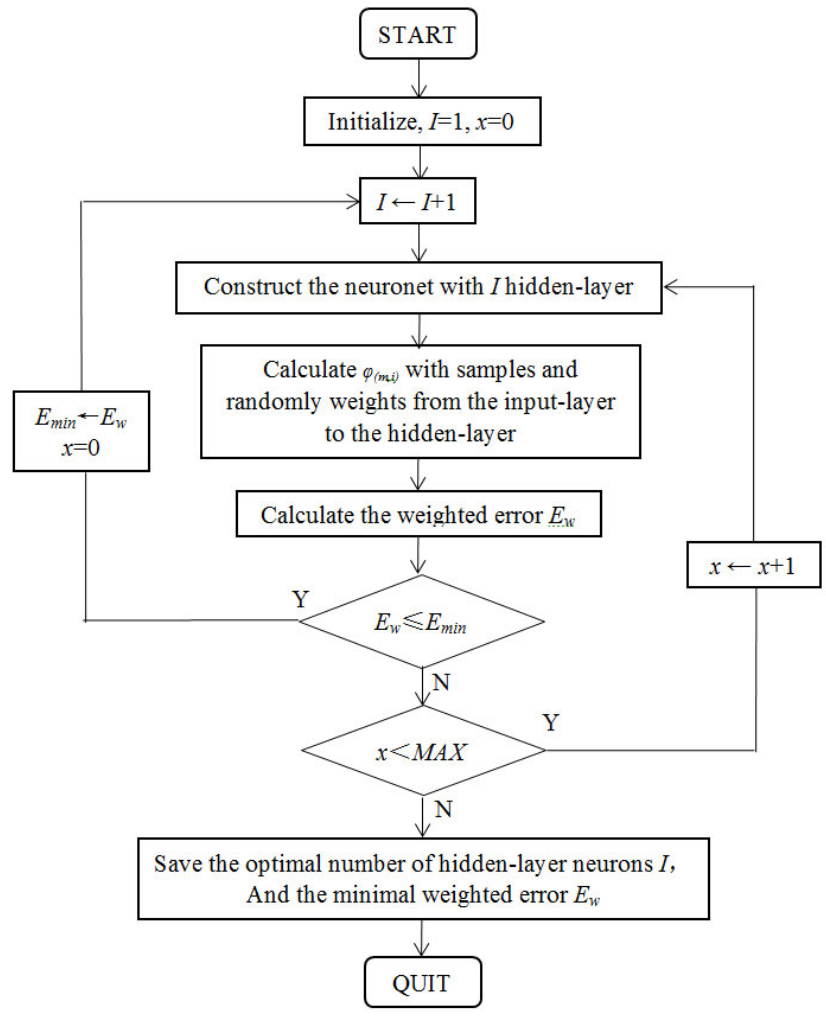

Figure 2. Flowchart of the WASD method
Data from 1952 to 1995 are used to learn. In order to improve the result, the data from 1996 to 2007 are used to verify.

It can be observed from Figure 2 that there are two conditions to control the structure of neuronet. According to the formula (4) (5), $E_{l}, E_{v}$ and $E_{w}$ can be figured out in the progress of training. $x<M A X$ can be recognized as the second condition to control whether to increase the number of hidden-layer nerves. Different MAX, such as 10, 100 or 1,000 , is chosen according to the stability of the net. In order to unify the result, MAX is set as 1,000 in this report.

$E_{w}$ is the crucial condition to change the hidden-layer nerves. The hidden-layer nerves are increased firstly. As soon as the minimal $E_{w}$ is found, reset the threshold value of hidden layer $\left(\beta_{i}\right)$ and the weights from the input layer to the hidden layer $\left(w_{i}\right)$. If the reset number of times comes up to the MAX value, the circulation is stopped and the $E_{w}$ is not changed.

\section{FORECAST HYDROPOWER GENERATION OF CHINA}

The minimal $E_{w}$ is the signal deciding whether to increase the hidden-layer neures, as is shown in Figure 3.

It has been shown that when the neurons increase to 6 , the weighted error and verified error are the smallest with the increasing hidden layer nerves. If nerves keep rising, the error 
will increase significantly. It can be concluded that, therefore, this value is the turning point and the optimal structure of neuronet is the hidden layer with six nerves.

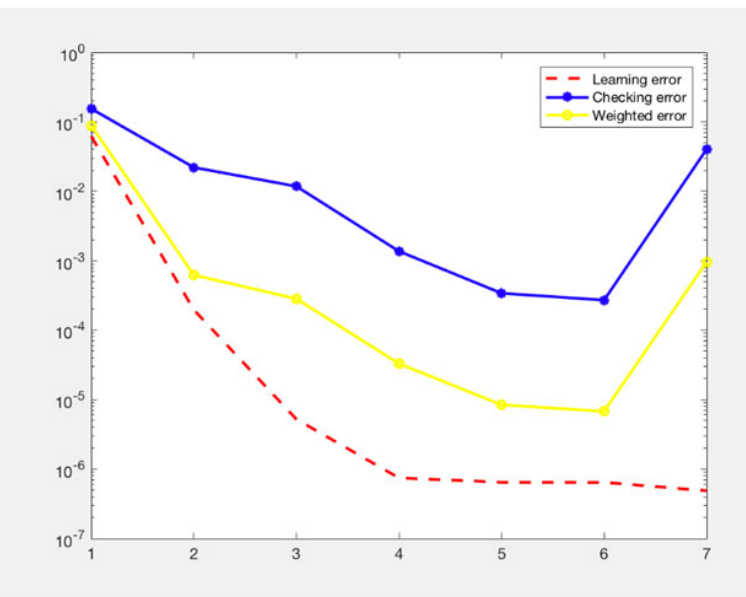

Figure 3. Mean square error and the hidden-layer neures

The data, which is from 1950 to 1995, in the first part is used to learn. The Figure 4 shows the effect of learning and the prediction to a large extent has large similarity to the actual data. Then, the verified data are used to make the prediction more precise.

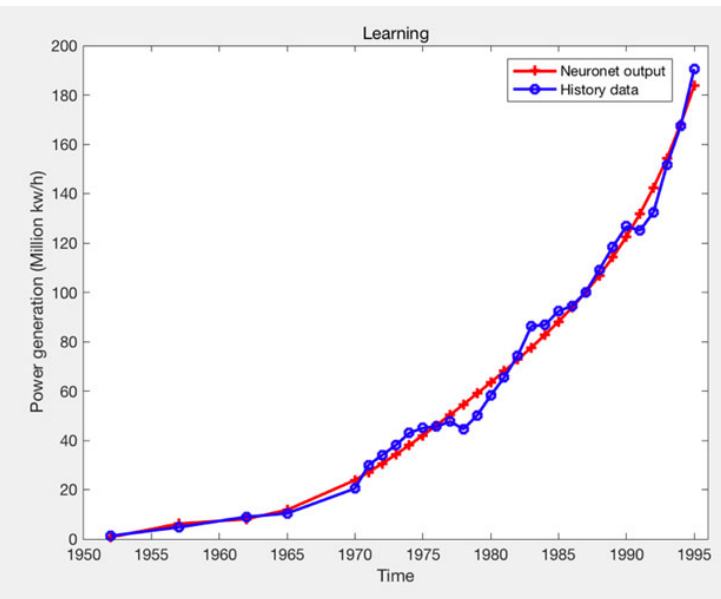

Figure 4. Learning effect of WASD neuronet

In Figure 5, the largest difference between prediction and actual data is 54.78 .

\section{Conclusion}

By using the existed neuronet with six hidden layers, the hydropower generation from 2018 to 2050 is predicted. From Figure 6 , there is a tiny difference between prediction and actual data from 2007 to 2017. From 2018 to 2050, the growing trend is sustainable.

Published by Sciedu Press

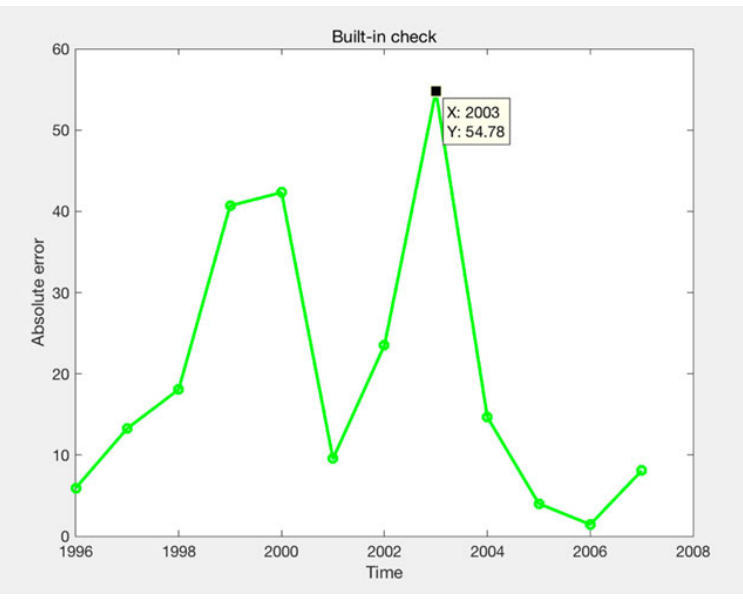

Figure 5. Absolute error in built-in check

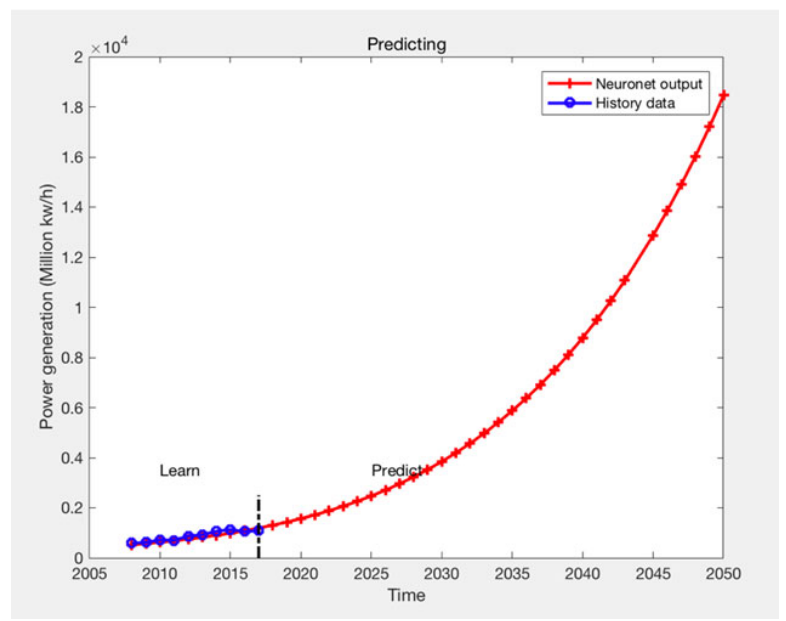

Figure 6. Prediction results synthesized by WASD neuronet

Actually, it is well known that water resource is not infinite. Without utilizing it reasonably, the generation would definitely fail to increase continuously. Because of the fact that the neuronet is only used to predict an ideal model of hydropower generation, in order to protect the natural environment, practical measures and further studies are significant and require seriously urgent considerations.

\section{ACKNOWLEDGeMents}

This work is supported by National Natural Science Foundation of China (No. 61703189), by the Opening Foundation of Key Laboratory of Opto-technology and Intelligent Control (Lanzhou Jiaotong University), Ministry of Education (No. KFKT2018-1), by the Belt and Road Special Project of Lanzhou University (No. 2018ldbryb020), by the Natural Science Foundation of Gansu Province, China (No. 18JR3RA264), by the Fundamental Research Funds for the Central Universities (No. lzujbky-2017-37), by the Natural Science Foundation of Hunan Province (No. 2017JJ3257), by the Research Foundation of Education Bureau of Hunan Province, China (No. 17C1299). 


\section{REFERENCES}

[1] Yetis Y, Jamshidi M. Forecasting of Turkey's electricity consumption using artificial neural network. 2014 World Automation Congress(WAC). https ://doi.org/10.1109/wac . 2014.69361 21

[2] Tang J, Cong Y, Gao HW. Prediction model design of short-term load for power system. Computer Simulation. 2016; 33(2): 171-174. ht tps://doi.org/10.3969/j.issn.1006-9348.2016.02.036

[3] Yi Z, Zhao Z. Electric power forecasting based on artificial neural network. Microprocessors. 2003; 0(4): 40-42. https ://doi.org/ 10.3969/j.issn.1002-2279.2003.04.015

[4] Wang DP. Research on grey prediction models and their applications in medium and long-term power load forecasting. Huazhong University of Science and Technology. 2013.

[5] Wang DD. Application research of gene expression programming in electricity load prediction. Hebei University of Engineering. 2013.

[6] Han FJ, Wang KF. The prediction of electricity consumption based on neural network-Markov model. Information Technology. https : //doi.org/10.13274/j.cnki.hdzj.2010.11.065

[7] Xin Y. Research on short term electric load prediction using kernel partial least square regression. Lanzhou Jiaotong University. 2017.

[8] Llanas B, Sainz FJ. Constructive approximate interpolation by neural networks. Journal of Computational and Applied Mathematics. 2005; 188(2): 283-308. https ://doi.org/10.1016/j.cam.2005.04 019

[9] Xiao L, Liao B L, Li S, et al. Nonlinear recurrent neural networks for finite-time solution of general time-varying linear matrix equations. Neural Networks. 2018. https://doi.org/10.1016/j.neunet .2017 .11 .011

[10] Lu YW, Sundararajan N, Saratchandran P. A sequential learning scheme for function approximation using minimal radial basis function neural networks. Neural Computation. 1997; 9(2): 461-478. https://doi.org/10.1162/neco.1997.9.2.461

[11] Mahil J, Raja TSR. An intelligent biological inspired evolutionary algorithm for the suppression of incubator interference in premature infants ECG. Soft Computing. 2014; 18(3): 571-578. http: //dx.doi.org/10.1007/s00500-013-1078-7

[12] John P, George L. Numerical methods using MATLAB. Computers \& Mathematics with Applications. 1996; 31(7): 147. https: //doi.org/10.1016/S0898-1221 (96)90473-3

[13] Jin L, Li S, La H, Luo X. Manipulability optimization of redundant manipulators using dynamic neural networks. IEEE Transactions on Industrial Electronics. 2017; 64(6): 4710-4720. https: //doi.org/10.1109/tie.2017.2674624

[14] Jin L, Zhang YN. Discrete-time Zhang neural network for online time-varying nonlinear optimization with application to manipulator motion generation. IEEE Transactions on Neural Networks and Learning Systems. 2015; 26(7): 1525-1531. https ://doi .org/10 $.1109 /$ tnnls. 2014.2342260

[15] Xiao L, Liao BL, Li S, et al. Design and Analysis of FTZNN Applied to Real-Time Solution of Nonstationary Lyapunov Equation and Tracking Control of Wheeled Mobile Manipulator. IEEE Transactions on Industrial Informatics. 2017. https ://doi.org/10.1 109/TII. 2017.2717020

[16] Guo DS, Zhang YN, He LY, et al. Chebyshev-polynomial neuronet, WASD algorithm and world population prediction from past 10000 year rough data. The 27th Chinese Control and Decision Conference (2015 CCDC). https : //doi .org/10.1109/ccdc. 2015.71 62194
[17] Zhang YN, Xiao ZL, Guo DS, et al. USPD doubling or declining in next decade estimated by WASD neuronet using data as of October 2013. Computational Intelligence and Intelligent Systems: 7th International Symposium, ISICA 2015. https://doi.org/10.1007/ 978-981-10-0356-1

[18] Mao MZ, Li J, Jin L, Li S, Zhang YN. Enhanced discrete-time Zhang neural network for time-variant matrix inversion in the presence of bias noises. Neurocomputing. 2016: 220-230. https : //doi .org/ 10.1016/j.neucom. 2016.05.010

[19] Li S, Zhang YN, Jin L. Kinematic control of redundant manipulators using neural networks. IEEE Transactions on Neural Networks and Learning Systems. 2017; 28(10): 2243-2254. https: //doi.org/10.1109/tnnls.2016.2574363

[20] Peng AJ, Yu G, Zeng H. Blind Median Filtering Detection Using Auto-Regressive Model and Markov Chain. International Journal of Robotics and Control. 2018; 1(1). https://doi.org/10.5430/ ijrc.vlnlp32

[21] Zhang YN, Yin YH, Guo DS, et al. Cross-validation based weights and structure determination of Chebyshev-polynomial neural networks for pattern classification. Pattern Recognition. 2014: 34143428. https://doi.org/10.1016/j.patcog.2014.04.026

[22] Zhang Y, Yu XT, Guo DS. Weights and structure determination of multiple-input feed-forward neural network activated by Chebyshev polynomials of class 2 via cross-validation. Neural Computing and Applications. 2014; 25(7-8): 1761-1770. https ://doi.org/10.1 007/s00521-014-1667-0

[23] Zhang YN, Yu XT, Guo DS, et al. Weights and structure determination of feed-forward two-input neural network activated by Chebyshev polynomials of class 2. 2012 24th Chinese Control and Decision Conference (CCDC). https://doi.org/10.1109/ccdc.2 012.6244175

[24] Jin L, Li S, Hu B. RNN Models for Dynamic Matrix Inversion: A Control-Theoretical Perspective. IEEE Transactions on Industrial Informatics. 2018; 14(1): 189-199. https ://doi.org/10.1109/ tii.2017.2717079

[25] Jin L, Li S, Luo X, Li Y, Qin B. Neural Dynamics for Cooperative Control of Redundant Robot Manipulators. IEEE Transactions on Industrial Informatics. 2018: 1-1. https : //doi .org/10.1109/ti i. 2018.2789438

[26] Jin L, Li S, Hu B. Noise-Suppressing Neural Algorithm for Solving Time-Varying System of Linear Equations: A Control-Based Approach. IEEE Transactions on Industrial Informatics. https : //doi.org/10.1109/tii.2018.2798642

[27] Jiang XY, Li S. Bas:beetle antennae search algorithm for optimization problems. International Journal of Robotics and Control. 2018; 1(1). https://doi.org/10.5430/ijrc.vlnlpl

[28] Ma C, Liao BL, Zhou J, et al. Prediction of Shanghai index based on the sine-activated WASD neural network. Journal of Huaihua University. 2017(11). https ://doi.org/10.3969/j.issn.167 1-9743.2017.11.020

[29] Zhang YN, Qu L, Liu JR, et al. Sine neural network with double-stage weights and structure determination. Soft Computing. 2012; 20(1): 211-221. https : //doi.org/10.1007/s00500-014-1491-6

[30] Zhang Y, Tan N. Weights direct determination of feedforward neural networks without iteration BP-training. Intelligent Soft Computation and Evolving Data Mining: Integrating Advanced Technologies. 2010: 220-248.

[31] Zhang YN, Yang YY, Li W. Weights direct determination of neural networks. Sun Yat-Sen University Press. 2010. 